\title{
Excitation of thin beams using asymmetric piezoelectric actuators ${ }^{\text {a) }}$
}

\author{
GaryP. Gibbs and Chris R. Fuller \\ Department of Mechanical Engineering, Virginia Polytechnic Institute and State University, Blacksburg, \\ Virginia 24061
}

(Received 20 September 1991; revised 29 April 1992; accepted 21 July 1992)

\begin{abstract}
In this paper, an approximate analytical model is developed for the excitation of a thin beam by a single piezoelectric actuator bonded to the surface of the beam. The premise of this work is to investigate the excitation of beams by piezoelectric actuators on a more fundamental level than present work, and then use the asymmetric model to predict a wave response, rather than a modal response, on more complicated structure/actuator systems. It is determined that the single surface mounted piezoelectric actuator simultaneously excites both flexural and extensional motion in beams whose relative amplitudes are functions of beam/actuator geometry and properties. The model is then applied to the excitation of an infinite beam by two colocated arbitrarily driven actuators. It is shown that this configuration can produce any desired combination of flexural and extensional waves in beams by varying the degree of asymmetry between the actuators.
\end{abstract}

PACS numbers: $43.40 . \mathrm{Cw}$, 43.88.Fx

\section{LIST OF SYMBOLS}

$A$ beam cross sectional area $\left(\mathrm{m}^{2}\right)$

$C \quad$ slope of the assumed strain distribution $\left(\mathrm{m}^{-1}\right)$

$d_{31} \quad$ strain sensitivity $\left(166 \times 10^{-12} \mathrm{~m} / \mathrm{V}\right.$ for piezoelectric G1195 material)

$E_{b} \quad$ Young's modulus of the beam ( $\left.\mathrm{Pa}\right)$

$E_{\mathrm{pe}} \quad$ Young's modulus of the piezoelectric material $(\mathrm{Pa})$

$E_{\text {eff }} \quad$ Effective Young's modulus of the beam $(\mathrm{Pa})$

$F_{w} \quad$ shear force $(N)$

$F_{x} \quad$ in-plane force $(N)$

$h$ half beam thickness (m)

$k_{e} \quad$ extensional wave number $\left(\mathrm{m}^{-1}\right)$

$k_{f} \quad$ flexural wave number $\left(\mathrm{m}^{-1}\right)$

$K^{e} \quad$ extensional excitation constant (dimensionless)

$K^{f} \quad$ flexural excitation constant $\left(\mathrm{m}^{-1}\right)$

$L \quad$ actuator length (m)

$M \quad$ internal bending moment $\left(\mathrm{N}^{*} \mathrm{~m}\right)$

$m, m(x) \quad$ actuator induced bending moment $\left(\mathrm{N}^{*} \mathrm{~m}\right)$

$T \quad$ actuator thickness (m)

$u(x) \quad$ unit step function (dimensionless)

$v_{w} \quad$ out-of-plane velocity $(\mathrm{m} / \mathrm{s})$ $v_{x} \quad$ in-plane velocity $(\mathrm{m} / \mathrm{s})$

$V \quad$ applied voltage (V)

$V_{1} \quad$ complex voltage applied to patch $1(\mathrm{~V})$

$V_{2} \quad$ complex voltage applied to patch 2 (V)

$w \quad$ beam displacement in $z$ direction $(\mathrm{m})$

$W \quad$ total beam displacement in $z$ direction (m)

$x \quad$ coordinate along beam neutral axis

$\boldsymbol{z}$

coordinate perpendicular to the beams surface

\section{Greek}

$\delta^{\prime} \quad$ derivative of the unit impulse function $\left(\mathrm{m}^{-2}\right)$

$\epsilon \quad$ strain (dimensionless)

$\epsilon_{0} \quad$ assumed strain at neutral axis (dimensionless)

$\rho \quad$ mass per unit volume of the beam $\left(\mathrm{kg} / \mathrm{m}^{3}\right)$

$\omega$ rotational frequency $(\mathrm{rad} / \mathrm{s})$

$\sigma_{b} \quad$ stress in beam $(\mathrm{Pa})$

$\sigma_{\mathrm{pe}} \quad$ stress in piezoelectric material $(\mathrm{Pa})$

$\zeta$

Z

$\Pi_{f}$

$\dot{\Pi}_{e}$

total beam displacement in $x$ direction ( $\mathrm{m}$ )

flexural power flow (W)

extensional power flow (W)

rotational velocity $(\mathrm{rad} / \mathrm{s})$

\section{INTRODUCTION}

Over the past 5 years, a significant amount of research has been dedicated to the use of piezoelectric actuators and sensors to control vibration in beam like structures. Early work by Crawley and de Luis ${ }^{1}$ demonstrated the effective use of piezoelectric actuators in controlling transient vibrations in beams. Both Fanson and Chen, ${ }^{2}$ and Crawley and Anderson ${ }^{3}$ have analyzed the forcing functions associated

\footnotetext{
a) Presented at the 121 st Meeting of the Acoustical Society of America, Baltimore, MD 29 April-3 May 1991 [J. Acoust. Soc. Am. 89, 1890(A) (1991)].
}

with two piezoelectric actuators bonded symmetrically on opposite sides of a thin beam. In their work, it is shown how the actuators, when driven with the same input voltage but $180 \mathrm{deg}$ out-of-phase, effectively exert equal and opposite line moments on the beam at the ends of the actuators and subsequently generate flexural motion in the beam. Crawley and Anderson ${ }^{3}$ have also shown that when the actuators are driven in-phase (symmetric excitation) the effective forcing functions are in-plane line forces of opposite direction, again concentrated at the ends of the actuator. The resulting motion in the beam in this case is purely extensional. 
Recently Clark et al. ${ }^{4}$ have experimentally and analytically studied the harmonic excitation of a simply supported beam using multiple pairs of piezoelectric actuators configured to excite pure flexure in the beam. Their work demonstrated that arrays of suitably driven actuators can be used to excite selected modes in the beam. The analytical model for the piezoelectric actuator forcing function, was also confirmed by the experimental results. Dimitriadis et al. ${ }^{5}$ have also extended the one-dimensional (1-D) analysis of Crawley and de Luis ${ }^{1}$ to excitation of thin plates by rectangular two-dimensional (2-D) piezoceramic patches. Their work showed that, for an infinitely thin bonding layer, the forcing function was a line moment around the complete periphery of the patch. This patch forcing function is the basis of analytical studies on active control of vibration and sound radiation from vibrating panels using piezoelectric actuators and sensors (see, for example, the work by Clark and Fuller ${ }^{6}$ ).

It is interesting to note that all of the previous work has been concerned with excitation and control of short finite beams dictating that the system response to the piezoelectric actuators is modally based. However, many structures in applications such as Naval vessels and aerospace structures are constructed from long slender beam elements in which the vibrational response is transmitted dominantly in wave type motion. Due to the inherent damping in these beams due to joints, attachments, etc., it is often inappropriate and unrealistic to use a modally based model. In connection with this, it has been demonstrated that long beams tend to transmit vibrational power in a wave flow type process and it is a better control goal to isolate or reduce the traveling energy rather than, say, the modal response. Experiments by Gibbs and Fuller ${ }^{7}$ have shown that narrow-band flexural power flow in thin semi-infinite beams can be reduced significantly by using a single pair of piezoelectric actuators configured to produce pure flexure. In order to analytically study long beam systems, it is thus necessary to derive the system response to the piezoelectric actuator from a wave rather than a modal approach. Note, however, that the two approaches are inherently related just as modes can be thought of as a superposition of opposite traveling waves of equal amplitudes.

A complete analysis/control of marine and aerospace type structures with long beam elements is further complicated by the presence of structural discontinuities such as joints, attachments, etc. Cremer et al. ${ }^{8}$ has shown that arbitrary boundary conditions at beam discontinuities can cause wave conversion between different forms (i.e., flexural, and extensional). As beams are significantly stiffer in extension than flexure, they may carry large vibrational energy in this form for relatively little detectable displacement. This inplane energy may subsequently be converted into other wave forms at discontinuities such as structural joints and reappear as large flexural motion. It is thus important to control all wave forms in complex vibrating structures to ensure global reductions. Experimental work by Fuller et al. ${ }^{9}$ has demonstrated that it is possible to simultaneously control extensional and flexural waves in semi-infinite beams using asymmetric pairs of piezoceramic actuators and sensors in conjunction with a two-channel adaptive LMS controller.
To analytically study such control configurations, it is thus necessary to derive the more general situation of excitation of thin beams by a single actuator bonded to the beam or pairs of asymmetrically arranged actuators. As noted above, all previous actuator models have been either for pure flexure or extension; no general model for asymmetric excitation is presently available.

It is thus the thrust of the paper to develop new analytical relations based on wave approaches for the excitation of thin long beams by a single piezoceramic element bonded to the surface (asymmetric excitation). The approach is to consider that the single piezoceramic actuator drives both extensional and flexural waves in the beam. The strain field under the piezoceramic element is thus, by superposition, the sum of the two wave components, and is coupled by the actuator. Once the waves leave the actuator location they are assumed to travel uncoupled in the beam. Similar approaches have been used in analysis of point force excitation of beams by forces that are not normal to the beam surface; ${ }^{10}$ the normal component of the force is considered to drive the flexural waves while the in-plane component drives extensional waves.

The analytical results can then be applied to more complicated arrays of actuators and sensors. As an example, the new analytical relations will then be used to study the excitation of infinite thin beams in terms of the resulting vibrational power flow in extension and/or flexure. The influence of relative actuator length and phase on flexural and extensional power flowing into the beam is also studied. The model can be used to study how flexural or extensional motion is effected by other actuator or beam parameters, and will be the basis for future analytical work concerned with multiwave control approaches.

\section{THEORETICAL DEVELOPMENT}

Figure 1 shows the strain distribution for pair of perfectly bonded piezoelectric actuators configured to excite either pure flexure of pure extension as analyzed by Crawley and Anderson. ${ }^{3}$ In the case of pure flexure, the finite patch effectively acts as equal and opposite line moments acting at its ends, and applied to the beam. In the case of pure extension, the finite patch effectively acts as equal and opposite in-plane point forces acting at the ends of the element on the neutral axes. In the following analysis, it will be shown how the asymmetrically bonded piezoelectric actuator simultaneously produces both extensional and flexural motion in thin beams and how their relative magnitudes are deter-

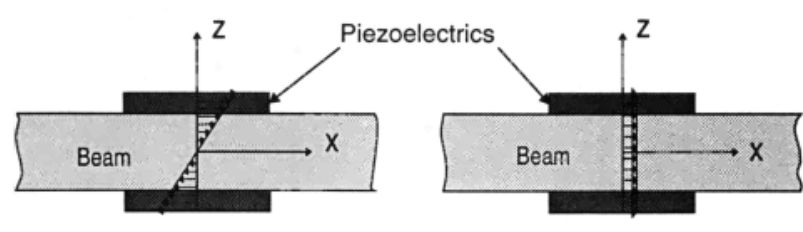

Flexural Strain Distribution

FIG. 1. Pure flexural/extensional strain distributions of symmetrically bonded piezoelectric actuators. 
mined by the actuator/beam configuration. The asymmetric actuator is thus assumed to be a superposition of the two strain distributions.

\section{A. Asymmetric plezoelectric element-infinite extent}

Piezoelectric elements are excited by applying an electrical voltage along their polarization direction. When the piezoelectric actuator is unconstrained, the actuator will strain an amount $\epsilon_{\mathrm{pe}}$ proportional to the applied voltage as shown in the following relation (where $d_{31}$ is the strain sensitivity of the material, $V$ is the excitation voltage, and $T$ is the material thickness):

$$
\epsilon_{\mathrm{pe}}=\left(d_{31} / T\right) V \text {. }
$$

For the purpose of this analysis, the single piezoelectric actuator is taken to be a piezoceramic G1195 material ${ }^{11}$ assumed to be perfectly bonded to the beam with zero glue layer thickness, as shown in Fig. 2 (top). The actuator is also assumed to be very long and thin compared to the beam thickness. The beam is infinite in the $x$ direction and has a height of $2 h$ in the $z$ direction. The beam has a modulus of elasticity $E_{b}$ and an area moment of inertia $I$.

It is assumed that a plane through the material perpendicular to the middle surface of the beam remains planar and perpendicular to the middle surface during deformation. This assumption is equivalent to ignoring shear strains in planes perpendicular to the middle surface of the beam, and is the basis of the Kirchhoff hypothesis of laminate-plate theory. ${ }^{12}$ When a voltage is applied across the transducer in the $z$ direction, the beam/piezoelectric element laminate will have an asymmetric strain distribution as shown in Fig. 2 (top) based on the assumptions of the Kirchhoff hypothesis.

The strain distribution in the $z$ direction, $\epsilon(z)$, within the cross section assuming an infinite extent piezoceramic element (i.e., no end effects) is shown in Fig. 2 (top) and can be written in the form shown in Eq. (2), where $C$ is the slope and $\epsilon_{0}$ is the $z$ intercept:

$$
\epsilon(z)=C z+\epsilon_{0} .
$$

Equation (2) can be decomposed into the sum of an antisymmetric distribution $C z$ (i.e., flexural component) about the center of the beam, and a uniform strain distribution $\epsilon_{0}$

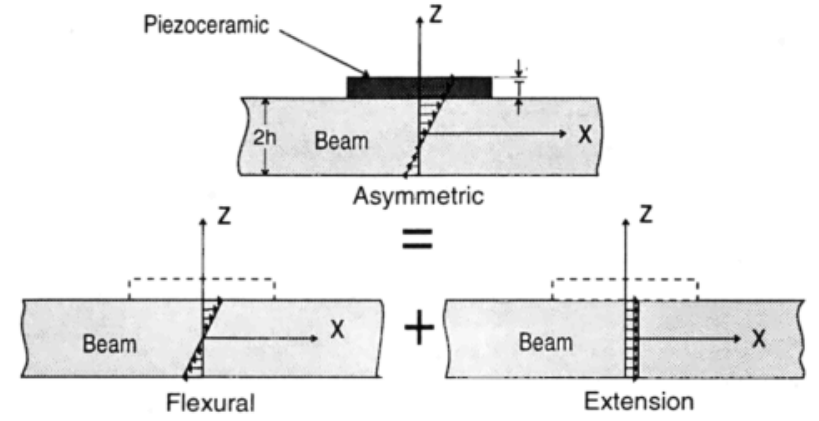

FIG. 2. Strain distribution of an asymmetric piezoelectric actuator.

(i.e., extensional component) as shown in Fig. 2.

Using the strain distribution of Fig. 2 (top) and Hooke's law, the stress distribution within the beam $\sigma_{b}(z)$ is given as follows:

$$
\sigma_{b}(z)=E_{b}\left(C z+\epsilon_{0}\right),
$$

where, $E_{b}$ is the Young's modulus of the beam. The stress distribution within the piezoceramic actuator $\sigma_{\mathrm{pe}}(z)$ is a function of the unconstrained piezoceramic actuator strain, Young's modulus of the piezoelectric actuator $E_{\mathrm{pe}}$, and the strain distribution shown in Fig. 2 (top) as follows:

$$
\sigma_{\mathrm{pe}}(z)=E_{\mathrm{pe}}\left(C z+\epsilon_{0}-\epsilon_{\mathrm{pe}}\right) .
$$

Applying moment equilibrium about the center of the beam produces the relation:

$$
\int_{-h}^{h} \sigma_{b}(z) z d z+\int_{h}^{h+T} \sigma_{\mathrm{pe}}(z) z d z=0 .
$$

Next applying force equilibrium in the $x$ direction is as follows:

$$
\int_{-h}^{h} \sigma_{b}(z) d z+\int_{h}^{h+T} \sigma_{\mathrm{pe}}(z) d z=0 .
$$

After integration equations (5) and (6) can be solved for the two unknowns, namely $\epsilon_{0}$ and $C$, which are presented (after some manipulation) as follows:

$$
\epsilon_{0}=K^{e} \epsilon_{\mathrm{pe}},
$$

where

$$
\begin{aligned}
K^{e}= & \frac{\left[T^{4} / 6+\left(4 E_{b} / 3 E_{\mathrm{pe}}\right) h^{3} T\right]}{\left[T^{4} / 6+\left(8 E_{b}^{2} / 3 E_{\mathrm{pe}}^{2}\right) h^{4}+\left(4 E_{b} / 3 E_{\mathrm{pe}}\right)\left(4 h^{3} T+3 h^{2} T^{2}+h T^{3}\right)\right]}, \\
C & =K^{f} \epsilon_{\mathrm{pe}},
\end{aligned}
$$

where

$$
K^{f}=\frac{T}{\left(2 h T+T^{2}\right)}\left(2-\frac{\left(\frac{2}{3} E_{b} E_{\mathrm{pe}} h T^{3}+\left(16 E_{b}^{2} / 3\right) h^{4}+\frac{1}{3} E_{\mathrm{pe}}^{2} T^{4}+\frac{8}{3} E_{b} E_{\mathrm{pe}} h^{3} T\right)}{\left(E_{\mathrm{pe}}^{2} T^{4} / 6+\frac{8}{3} E_{b}^{2} h^{4}+\frac{4}{3} E_{b} E_{\mathrm{pe}}\left(4 h^{3} T+3 h^{2} T^{2}+h T^{3}\right)\right)}\right)
$$

The general equation of motion of a solid medium in terms of motion in all three coordinate directions is inherently coupled. ${ }^{8}$ However, as stated in Ref. 8, if a beam's thickness is less than a quarter wavelength in the direction of propaga- tion, then the beam motion can be accurately separated into its out-of-plane (flexural) motion and its in-plane (extensional) motion.

The next step is to separate out the flexural and exten- 
sional components. The antisymmetric portion of the strain distribution will induce bending moments in the beam and will subsequently drive flexural waves. The induced moment distribution in the beam in the proximity of the actuator, $m(x)$, is equal to the product of the bending stiffness, $E_{b} I$, times the slope $C$ :

$$
m(x)=E_{b} I C=E_{b} / K^{f} \epsilon_{\mathrm{pe}} .
$$

The uniform strain distribution portion $\epsilon_{0}$ will excite purely extensional waves. The uniform strain across the section $\epsilon(x)$ is also constant in the $x$ direction and equal to the following:

$$
\epsilon(x)=\epsilon_{0}=K^{e} \epsilon_{\mathrm{pe}} .
$$

\section{B. Flexural equation of motion}

The Euler equation of motion for flexural vibration of the thin beam is given as follows: ${ }^{8}$

$$
\partial^{2} \frac{\left[M_{x}-m(x)\right]}{\partial x^{2}}-\omega^{2} \rho A w=0,
$$

where $M_{x}$ is the internal bending moment within the beam and $m(x)$ is the actuator induced bending moment. Following the approach of Crawley, Eq. (9) can be modified for a finite length patch of length $L$, and then substituted into Eq. (11). For a finite length element, Eq. (9) is written as follows:

$$
m(x)=E_{b} I K^{f} \epsilon_{\mathrm{pe}}[u(x)-u(x-L)],
$$

where $u()$ is the unit step function.

The moment distribution is substituted into Eq. (11) and the second partial derivative with respect to $x$ is then taken. The result is brought to the right-hand side of the equation as shown:

$$
\frac{\partial^{2} M_{x}}{\partial x^{2}}-\omega^{2} \rho A w=E_{b} I K^{f} \epsilon_{\mathrm{pe}}\left[\delta^{\prime}(x)-\delta^{\prime}(x-L)\right] .
$$

The right-hand side is equivalent to equal and opposite line moments, proportional to $\epsilon_{\mathrm{pe}}$ [where $\epsilon_{\mathrm{pe}}$ is related to the applied voltage by Eq. (1)], and acting at the ends of the element. Equation (13) is the equation of motion for flexural excitation by a single asymmetric piezoelectric actuator. This equation can be applied to a thin beam subjected to arbitrary boundary conditions whether the solution is found using a modal or wave approach.

\section{Extensional equation of motion}

The equation of motion for extensional vibration is given as follows: ${ }^{8}$

$$
\frac{d^{2} \zeta}{d x^{2}}+\frac{\omega^{2} \rho}{E_{\text {eff }}} \xi=\frac{d \epsilon(x)}{d x},
$$

where $\zeta$ is the in plane beam displacement, $\rho$ is the density, and $\epsilon(x)$ is the actuator induced in plane strain distribution. Equation (10) can be thus modified for a finite length patch by assuming excitation strain only under the element as follows:

$$
\epsilon(x)=K^{e} \epsilon_{\mathrm{pe}}[u(x)-u(x-L)]
$$

Equation (15) is then substituted into Eq. (14), which produces the following nonhomogenous equation of motion:

$$
\frac{d^{2} \zeta}{d x^{2}}+\frac{\omega^{2} \rho}{E_{\text {eff }}} \xi=K^{e} \epsilon_{\mathrm{pe}}[\delta(x)-\delta(x-L)] .
$$

In this case, the finite element can be seen to be equivalent to two equal and opposite line forces acting in the $x$ direction at the ends of the element. Equation (16) is the equation of motion for extensional excitation by a single asymmetric piezoelectric actuator. This equation can also be applied to a thin beam subjected to arbitrary boundary conditions whether the solution is found using a modal or wave approach.

\section{Application to infinite beam power flow}

Simultaneously controlling both flexural and extensional motion in beams has been shown to be an important problem. ${ }^{7}$ In order to efficiently control these types of vibration, it is important to be able to effectively drive both forms of motion or waves with a single pair of actuators.

In order to demonstrate the usefulness of the asymmetric beam excitation, an infinite beam will be analyzed that has two symmetrically bonded finite piezoceramic actuators as shown in Fig. 3. The actuators are located with one edge at $x=0$ and have a length $L$. Each piezoceramic will be treated as a single independent actuator with variable relative complex amplitude in order to study how parameters such as length, relative phase and frequency effect the power flow of the subsequent flexural and extensional waves. Note in this case, since the beam is infinite, waves will be generated in contrast to the modal response of a finite structure. In the previous work, the response of the beam structures to excitation by piezoelectric elements was primarily from a modal approach not a wave approach, however, the two situations are inherently related. It is believed that the wave approach allows a better understanding of the coupling between the actuator and beam as it is less restricted by boundary conditions. The following analysis assumes continuity of strain energy from under the actuator to the infinite beam interface.

The flexural equation of motion, Eq. (13), is solved for each individual element (with excitation voltages $V_{1}$ and $\left.V_{2}\right)$, and the motion, $W(x, t)$, for the two collocated elements is found by superposition to be (as derived in Appen$\operatorname{dix} \mathbf{A})$ :

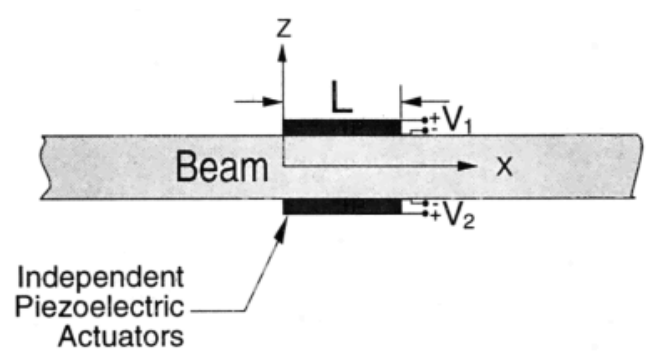

FIG. 3. Infinite beam arrangement with two actuators. 


$$
\begin{aligned}
W(x, t)= & \frac{d_{31} K^{f}\left(V_{1}-V_{2}\right)}{4 k_{f}^{2} T} \\
& \times\left[\left(1-e^{k_{\rho} L}\right) e^{-k_{f} x}-\left(1-e^{-j k_{f} L}\right) e^{j k_{\rho} x}\right] e^{-j \omega t},
\end{aligned}
$$

where $\omega$ is the rotational frequency, and $k_{f}$ is the flexural wave number. The power flow for the flexural wave is found by taking the sum of the time-averaged dot product of the shear force with the velocity, and the bending moment with the rotational velocity. ${ }^{8}$ As derived in Appendix B, the power flow $\Pi_{f}(\omega)$ at the frequency of interest is equal to:

$$
\Pi_{f}(\omega)=\frac{E_{b} I\left(K^{b}\right)^{2} d_{31}^{2} \omega}{8 T^{2} k_{f}}\left|V_{1}-V_{2}\right|^{2}\left[1-\cos \left(k_{f} L\right)\right] .
$$

The extensional equation of motion, Eq. (16), is solved as shown in Appendix A for one element and the displacement, $Z(x, t)$, for two collocated elements is found by superposition to be

$$
Z(x, t)=-\frac{j K^{e} d_{31}\left(V_{1}+V_{2}\right)}{2 k_{e} T}\left(1-e^{-j k_{e} L}\right) e^{j k_{e} x} e^{-j \omega t},
$$

where $k_{e}$ is the extensional wave number. The power flow for the extensional wave is found by taking the time-averaged dot product of the longitudinal force with the longitudinal velocity. ${ }^{8}$ As outlined in Appendix B the power flow, $\Pi_{e}(\omega)$, for the frequency of interest is found to be

$$
\begin{aligned}
\Pi_{e}(\omega)= & \frac{\left(K^{e}\right)^{2} d_{31}^{2} \omega E_{\mathrm{eff}} A}{4 k_{e} t^{2}} \\
& \times\left|V_{1}+V_{2}\right|^{2}\left[1-\cos \left(k_{e} L\right)\right] .
\end{aligned}
$$

It is interesting to note that in the previous work two pairs of actuators and sensors would have been necessary to excite both forms of motion; one dedicated to purely flexural, and the other dedicated to purely extensional motion. As will be shown in the next section, simultaneous excitation of both flexural and extensional motion of arbitrary complex amplitudes can be accomplished with a single actuator pair.

\section{RESULTS}

A computer algorithm was written to demonstrate asymmetric excitation of an infinite thin beam. The results derived show how both the flexural and extensional power flow in the infinite beam [Eqs. (18) and (20)] varies as a function of the magnitude and phase of the inputs $V_{1}$ and $V_{2}$ (i.e., the degree of asymmetry), as well as the actuator length $L$. The beam for this simulation was considered to be made of standard aluminum and had a height $2 h$ equal to $3.175 \mathrm{~mm}$ and a width equal to $7.62 \mathrm{~cm}$.

For the first result the excitation frequency of the piezoceramic actuators is $800 \mathrm{~Hz}$. The length of the piezoceramic actuators is taken as $3.81 \mathrm{~cm}$. The magnitude of $V_{1}$ and $V_{2}$ is fixed at $400 \mathrm{~V}$ peak to peak. These values are representative of typical applications. The flexural and extensional power flow ( $\mathrm{dB}$ relative to $10^{-12} \mathrm{~W}$ ) is plotted as a function of the phase between $V_{1}$ and $V_{2}$ in Fig. 4 . It has been previously

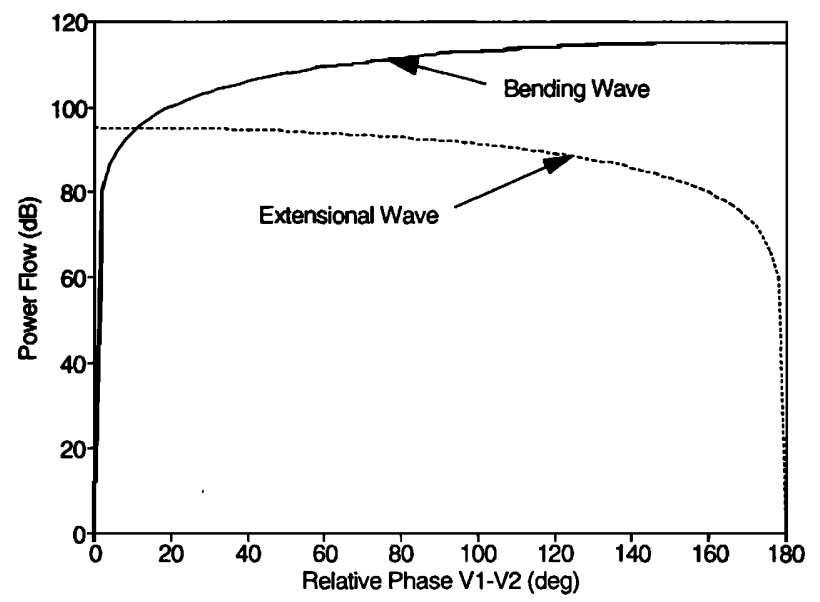

FIG. 4. Infinite beam power flow, $800 \mathrm{~Hz}, L=3.81 \mathrm{~cm}$.

stated that when both of the piezoceramics are driven in phase only an extensional wave is generated and correspondingly only extensional power flow is seen in Fig. 4 at this phase angle. When the piezoceramics are driven $180 \mathrm{deg}$ out of phase only a flexural wave is produced as can be seen in the figure. For phase angles between these ranges, both extensional and flexural waves can be driven simultaneously. The relative magnitude can be determined by choosing the appropriate phase from Fig. 4.

In the second illustrative result, the excitation frequency is $800 \mathrm{~Hz}$ and the magnitudes of the input voltages are again set equal to $400 \mathrm{~V}$ peak to peak. The phase of $V_{1}$ is chosen as $90 \mathrm{deg}$ ahead of $V_{2}$ such that both types of motion are present. The power flow for both the extensional and flexural waves as a function of actuator length is presented in Fig. 5. It can be seen that as the actuator length approaches zero the power flow for both wave types approaches zero. When the actuator length is equal to $9.6 \mathrm{~cm}$ (corresponding to half a flexural wavelength at this frequency) the bending wave is at a maximum because, as shown previously, an individual piezoceramic actuator effectively acts as two opposite phase moment sources externally applied to the beam at the

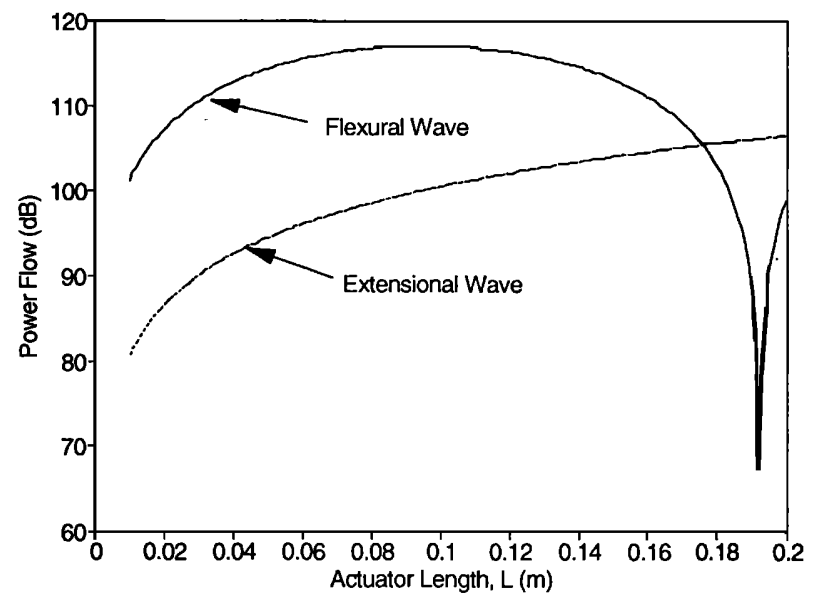

FIG. 5. Infinite beam power flow, $800 \mathrm{~Hz}$, relative phase $=90 \mathrm{deg}$. 
element ends. The waves generated at each end are thus perfectly in phase. When the actuator is $19.2 \mathrm{~cm}$ long, the length corresponds to a flexural wavelength, and the flexural power flow is a minimum (equal to zero) because the two opposite moment inputs produce wave fields which when superimposed produce zero displacement in the far field. The results imply that there is an optimal length actuator for each frequency of excitation.

The above conclusions can be extended to the response of a system from a modal point of view, as described in the following simple example. If an actuator pair, configured for pure flexure, completely covers the length of a simply supported beam, then the actuator length will be equal to a flexural wavelength for the second mode of vibration. The above wave point of view predicts the second mode would not be excited. The corresponding modal point of view is that while the actuator forcing function is uniform over the beam length, the second mode has equal lengths of positive and negative strain distributions and thus will not be excited. This simple example illustrates the equivalence of a wave and modal approach in terms of a finite beam response. The wave approach, however, is more fundamental in that it can be applied to infinite structures and can be extended to include evanescent near fields for flexural motion.

From the results, it is also apparent that the extensional wave does not have a maximum power flow until the actuator length is significantly increased. This behavior is due to the much higher phase speed of the extensional wave. The far-field power flow is a maximum when the actuator is a half-wavelength $(3.1 \mathrm{~m})$, but this is impractical with the current size availability of piezoceramic actuators. It can be seen that if extensional motion is desired, then it is more efficient to make the actuator as long as possible.

\section{CONCLUSIONS}

An approximate analysis of excitation of a thin beam by an asymmetric piezoceramic element is presented for the first time. The analysis shows that the single actuator is mathematically equivalent to equal and opposite moments (i.e., the $\delta^{\prime}$ functions in the out-of-plane force equation ), and simultaneously equal and opposite in-plane forces ( $\delta$ functions in the in-plane displacement equation) located at the ends of the actuator. The relative magnitudes of these forces/moments are shown to be dependent upon the actuator asymmetry and can be controlled by using a pair of collocated actuators with independent input voltage magnitudes and phases.

The model is then used to study excitation of both flexural and extensional waves in beams for various degrees of asymmetry and actuator length. The representative structure in the example is an infinite beam, but the analysis can be equally applied to relatively long beamlike structures or finite structures. The work presented here represents an important step because it represents the analytical basis by which simultaneous active control of both flexural and extensional motion in flexible structures can be studied, and optimized.

\section{ACKNOWLEDGMENTS}

The authors wish to express their appreciation to Rich Silcox and Harold Lester of the Structural Acoustics Branch of NASA Langley Research Center for their assistance in this project. The work was supported by the Structural Acoustics Branch of the NASA Langley Research Center under Grant No. NAG-1-390.

\section{APPENDIX A: SOLUTIONS TO THE FLEXURAL AND EXTENSIONAL EQUATIONS OF MOTION}

As presented in the paper, the equation of motion for flexural excitation by a single piezoelectric element is

$$
\frac{\partial^{2} M_{x}}{\partial x^{2}}-\omega^{2} \rho A w=E_{b} I K^{f} \epsilon_{\mathrm{pe}}\left[\delta^{\prime}(x)-\delta^{\prime}(x-L)\right] .
$$

Equation (A1) is solved by taking the Fourier transform of both sides and the result is presented as follows:

$$
E I v^{4} \widehat{w}(v)-\omega^{2} \rho A \widehat{w}(v)=j v E_{b} I K^{f} \epsilon_{\mathrm{pe}}\left(1-e^{-j v L}\right) .
$$

Equation (A2) is solved for $w(v)$ and then the inverse Fourier transform is applied to find $w(x)$, which is presented as follows:

$$
\begin{aligned}
w(x)= & \frac{d_{31} K^{f} V}{4 k_{f}^{2} T} \\
& \times\left[\left(1-e^{k_{f} L}\right) e^{-k_{f} x}-\left(1-e^{-j k_{f} L}\right) e^{j k_{\rho} x}\right] .
\end{aligned}
$$

For the case where two elements are collocated axially on opposite sides of the beam the total solution is found to be

$$
\begin{aligned}
W(x, t)= & \frac{d_{31} K^{f}\left(V_{1}-V_{2}\right)}{4 k_{f}^{2} T} \\
& \times\left[\left(1-e^{k_{f} L}\right) e^{-k_{\rho} x}-\left(1-e^{-j k_{f} L}\right) e^{j k_{f} x}\right] e^{-j \omega t} .
\end{aligned}
$$

The equation of motion for extensional excitation by one piezoelectric element as presented in the paper is as follows:

$$
\frac{d^{2} \zeta}{d x^{2}}+\frac{\omega^{2} \rho}{E_{\mathrm{eff}}} \zeta=K^{e} \epsilon_{\mathrm{pe}}[\delta(x)-\delta(x-L)] .
$$

Equation (A6) is solved by taking the Fourier transform of both sides and the result is presented as follows:

$$
-v^{2} \hat{\zeta}(v)+\frac{\omega^{2} \rho}{E_{\mathrm{eff}}} \hat{\zeta}(v)=K^{e} \epsilon_{\mathrm{pe}}\left(1-e^{-j v L}\right) .
$$

Equation (A7) is solved for $\zeta(v)$ and then the inverse Fourier transform is applied to find $\zeta(x)$, which is presented as follows:

$$
\zeta(x)=-\frac{j K^{e} d_{31} V}{2 k_{e} T}\left(1-e^{-j k_{e} L}\right) e^{j k_{e} x} .
$$

For the case where two elements are collocated on opposite sides of the beam the total solution is found to be 


$$
Z(x, t)=\frac{-j K^{e} d_{31}\left(V_{1}+V_{2}\right)}{2 k_{e} T}\left(1-e^{-j k_{e} L}\right) e^{j k_{e} x} e^{-j \omega t} .
$$

\section{APPENDIX B: DERIVATION OF FAR-FIELD POWER FLOW FOR FLEXURAL AND EXTENSIONAL EXCITATION}

The total response for flexural excitation of a thin beam by two axially colocated actuators bonded symmetrically on opposite sides of the beam is

$$
\begin{aligned}
W(x, t)= & \frac{d_{31} K^{f}\left(V_{1}-V_{2}\right)}{4 k_{f}^{2} t} \\
& \times\left[\left(1-e^{k_{f} L}\right) e^{-k_{f} x}-\left(1-e^{-j k_{f} L}\right) e^{j k_{f} x}\right] e^{-j \omega t} .
\end{aligned}
$$

The power flow due to the flexural excitation is found by the following relation: ${ }^{8}$

$$
\Pi_{f}(\omega)=\frac{1}{2} \operatorname{Re}\left(F_{w} V_{w}^{*}+M \dot{\Theta}^{*}\right),
$$

where $F_{w}$ is the shear force, $v_{w}$ is the out-of-plane velocity, $M$ is the bending moment, and $\Theta$ is the rotational velocity given by

$$
\begin{aligned}
v_{w}=\frac{\partial w}{\partial t}= & -\frac{j \omega d_{31} K^{f}\left(V_{1}-V_{2}\right)}{4 k_{f}^{2} T} \\
& \times\left[\left(1-e^{k_{f} L}\right) e^{-k_{f} x}-\left(1-e^{-j k_{f} L}\right) e^{j k_{f} x}\right] e^{-j \omega t},
\end{aligned}
$$$$
F_{w}=E_{b} I \frac{\partial^{3} w}{\partial x^{3}}=\frac{E_{b} I k_{f} d_{31} K^{f}\left(V_{1}-V_{2}\right)}{4 T}
$$

$$
\begin{gathered}
\times\left[-\left(1-e^{k_{f} L}\right) e^{-k_{\rho} x}\right. \\
\left.+j\left(1-e^{-j k_{f} L}\right) e^{j k_{\rho} x}\right] e^{-j \omega t}, \\
\dot{\theta}=\frac{\partial v_{w}}{\partial x}=\frac{\omega d_{31} K^{f}\left(V_{1}-V_{2}\right)}{4 k_{f} T}\left[j\left(1-e^{k_{f} L}\right) e^{-k_{\rho} x}\right. \\
\left.-\left(1-e^{-j k_{f} L}\right) e^{j k_{\rho} x}\right] e^{-j \omega t}, \\
M=-E_{b} I \frac{\partial^{2} w}{\partial x^{2}}=\frac{E_{b} I d_{31} K^{f}\left(V_{1}-V_{2}\right)}{4 T} \\
\times\left[-\left(1-e^{k_{f} L}\right) e^{-k_{\rho} x}\right. \\
\left.-\left(1-e^{-j k_{\mu} L}\right) e^{j k_{\rho} x}\right] e^{-j \omega t} .
\end{gathered}
$$

The argument of Eq. (B2), namely $F_{w} V_{w}^{*}+M \Theta^{*}$, after Eqs. (B3)-(B6) are substituted is presented as follows:

$$
\begin{aligned}
F_{w} V_{w}^{*}+M \dot{\Theta} *= & \frac{E_{b} I d_{31}^{2}\left(K^{f}\right)^{2} \omega}{8 T^{2} k_{f}}\left|V_{1}-V_{2}\right|^{2}\left(j\left(1-e^{k_{f} L}\right)\right. \\
& \times\left\{\cos \left(k_{f} x\right)-\cos \left[k_{f}(x-L)\right]\right. \\
& \left.-\sin \left(k_{f} x\right)+\sin \left[k_{f}(x-L)\right]\right\} e^{-k_{f} x} \\
& \left.+\left[2-2 \cos \left(k_{f} L\right)\right]\right) .
\end{aligned}
$$

It is interesting to note that in the above relation the near fields associated with the actuators only affect reactive energy, and are thus not part of the real power flow. When Eq. (B7) is further reduced to the form of Eq. (B2), the resulting flexural power flow is

$$
\Pi_{f}(\omega)=\frac{E_{b} I\left(K^{f}\right)^{2} d_{31}^{2} \omega}{8 T^{2} k_{f}}\left|V_{1}-V_{2}\right|^{2}\left[1-\cos \left(k_{f} L\right)\right]
$$

The total response for the extensional excitation of a thin beam by two axially colocated actuators bonded symmetrically on opposite sides of the beam is

$$
Z(x, t)=\frac{-j K^{e} d_{31}\left(V_{1}+V_{2}\right)}{2 k_{e} T}\left(1-e^{-j k \mu}\right) e^{j k x} e^{-j \omega t} .
$$

The power flow due to extensional excitation is found by the following relation:

$$
\Pi_{e}(\omega)=\frac{1}{2} \operatorname{Re}\left(F_{x} V_{x}^{*}\right),
$$

where $F_{x}$ is the in-plane force and $v_{x}$ is the in-plane velocity given by

$$
\begin{aligned}
& F_{x}=-E A \frac{\partial Z}{\partial x}= \\
& \quad-\frac{E_{\text {eff }} A K^{e} d_{31}\left(V_{1}+V_{2}\right)}{2 T} \\
& \times\left(1-e^{-j k_{e} L}\right) e^{j k_{x} x} e^{-j \omega t}, \\
& v_{x}=\frac{\partial Z}{\partial t}=-\frac{\omega K^{e} d_{31}\left(V_{1}+V_{2}\right)}{2 k_{e} T} \\
& \\
& \times\left(1-e^{-j k_{e} L}\right) e^{j k_{e} x} e^{-j \omega t} .
\end{aligned}
$$

When Eqs. (B10) and (B11) are substituted into (B9) the resulting extensional power flow is

$$
\Pi_{e}(\omega)=\frac{\left(K^{e}\right)^{2} d_{31}^{2} \omega E_{\text {eff }} A}{4 k_{e} T^{2}}\left|V_{1}+V_{2}\right|^{2}\left[1-\cos \left(k_{e} L\right)\right] .
$$

1 E. F. Crawley and J. de Luis, "Use of Piezoelectric Actuators as Elements of Intelligent Structures," AIAA J. 25, 1373-1385 (1987).

${ }^{2}$ J. L. Fanson, and J. C. Chen, "Structural Control by the Use of Piezoelectric Active Members," in Proceedings of NASA / DOD Control-Structures Interaction Conference, Rep. No. NASA CP-2447 (1986), Pt. II.

${ }^{3}$ E. F. Crawley and E. H. Anderson, "Detailed Models of Piezoceramic Actuation of Beams," AIAA Paper 89-1388-CP (1989).

${ }^{4}$ R. L. Clark, C. R. Fuller, and A. Wicks, "Characterization of Multiple Piezoelectric Actuators for Structural Excitation,” J. Acoust. Soc. Am. 90, 346-357 (1991).

${ }^{5}$ E. K. Dimitriadis, C. R. Fuller, and C. A. Rogers, "Piezoelectric Actuators for Distributed Vibration Excitation of Thin Plates," ASME J. Vib. Acoust. 113, 100-107 (1991).

${ }^{6}$ R. L. Clark and C. R. Fuller, "Control of Sound Radiation with Adaptive Structures," J. Intell. Mat. Systems Struct. 2(3), 431-452 (1991).

${ }^{7}$ G. P. Gibbs and C. R. Fuller, "Experiments on Active Control of Vibrational Power Flow Using Piezoceramic Actuators and Sensors," AIAA J. 30(2), 457-463 (1992).

${ }^{8}$ L. Cremer, M. Heckel, and C. A. Ungar, Structure-Borne Sound (Springer-Verlag, Berlin, 1973).

${ }^{9}$ C. R. Fuller, G. P. Gibbs, and R. J. Silcox, "Simultaneous Active Control of Flexural and Extensional Power Flow in Beams," J. Intell. Mat. Systems Struct. 1 (2), 235-247 (April 1990).

${ }^{10} \mathrm{~J}$. Pan and C. H. Hansen, "Active Control of Total Power Flow along a Beam," Proceedings from the International Congress on Recent Developments in Air and Structure-Borne Sound and Vibration, Auburn University, AL (6-8 March 1990).

${ }^{11}$ Piezo Systems, Piezoelectric Motor/Actuator Kit Manual, Piezoelectric Products, Inc., Advanced Technology Group, Cambridge, MD (1987).

${ }^{12}$ R. M. Jones, Mechanics of Composite Materials (Scripta, Washington, DC, 1975). 